\title{
Genotyping of bacteria belonging to the former Erwinia genus by PCR-RFLP analysis of a recA gene fragment
}

\author{
Małgorzata Waleron, Krzysztof Waleron, Anna J. Podhajska \\ and Ewa Łojkowska
}

Department of

Biotechnology,

Intercollegiate Faculty of

Biotechnology, University of

Gdansk and Medical

University of Gdansk, Kładki

24, 80-822 Gdansk, Poland
Author for correspondence: Ewa Lojkowska. Tel: +4858 32022 48. Fax: +48 583012807. e-mail: lojkowsk@biotech.univ.gda.pl

Genotypic characterization, based on the analysis of restriction fragment length polymorphism of the recA gene fragment PCR product (recA PCR-RFLP), was performed on members of the former Erwinia genus. PCR primers deduced from published recA gene sequences of Erwinia carotovora allowed the amplification of an approximately 730 bp DNA fragment from each of the 19 Erwinia species tested. Amplified recA fragments were compared using RFLP analysis with four endonucleases (Alul, Hinfl, Tasl and Tru1I), allowing the detection of characteristic patterns of RFLP products for most of the Erwinia species. Between one and three specific RFLP groups were identified among most of the species tested (Erwinia amylovora, Erwinia ananas, Erwinia cacticida, Erwinia cypripedii, Erwinia herbicola, Erwinia mallotivora, Erwinia milletiae, Erwinia nigrifluens, Erwinia persicina, Erwinia psidii, Erwinia quercina, Erwinia rhapontici, Erwinia rubrifaciens, Erwinia salicis, Erwinia stewartii, Erwinia tracheiphila, Erwinia uredovora, Erwinia carotovora subsp. atroseptica, Erwinia carotovora subsp. betavasculorum, Erwinia carotovora subsp. odorifera and Erwinia carotovora subsp. wasabiae). However, in two cases, Erwinia chrysanthemi and Erwinia carotovora subsp. carotovora, 15 and 18 specific RFLP groups were detected, respectively. The variability of genetic patterns within these bacteria could be explained in terms of their geographic origin and/or wide host-range. The results indicated that PCR-RFLP analysis of the recA gene fragment is a useful tool for identification of species and subspecies belonging to the former Erwinia genus, as well as for differentiation of strains within $E$. carotovora subsp. carotovora and $E$. chrysanthemi.

Keywords: fingerprinting, differentiation, plant-pathogenic bacteria, recombinase A

\section{INTRODUCTION}

Several Gram-negative, non-spore-forming, facultatively anaerobic, rod-shaped bacteria have been classified traditionally into the genus Erwinia, mainly on the basis of their association with plants as either pathogens, epiphytes or saprophytes (Lelliott \& Dickey, 1984). The species status is usually defined by biochemical and physiological tests (Verdonck et al., 1987). Plant pathologists have divided the strains into three groups based on numerical taxonomic analysis (Dye, 1968, 1969a, b, c). The 'carotovora' group is characterized by the ability to produce pectinolytic enzymes causing soft rot. The 'amylovora' group consists of pathogens that cause dry necrotic or wilting symptoms on their host plants. The 'herbicola' group consists of epiphytes, weak plant pathogens and saprophytes occurring in soil, water or the atmosphere, as well as in animal or human tissues. In the latter case they are classified in the genus Enterobacter (Beji et al., 1988; Lind \& Ursing 1986).

Several immunological methods have been developed for the detection and identification of Erwinia carotovora subsp. atroseptica (De Boer \& McNaughton; 1987; Gorris et al., 1994; Hyman et al., 1995), Erwinia chrysanthemi (Samson et al., 1989) and Erwinia amylovora (Vantomme et al., 1982). Furthermore, tests based on PCR have been described only for the more econ- 
omically important Erwinia species: E. carotovora subsp. atroseptica (Darrasse et al., 1994; De Boer \& Ward, 1995; Fréchon et al., 1998; Smid et al., 1995), E. amylovora (Bereswill et al., 1992, 1995; Guilford et al., 1996; McManus \& Jones, 1995), E. chrysanthemi (Nassar et al., 1996) and Ligase Chain Reaction (LCR) for Erwinia stewartii (Willson et al., 1994). Toth et al. (1999a) described a one-step 16S rDNA PCR-based method for the detection of all soft rot Erwinia species. However, this method did not enable the identification of the species and subspecies within the genus Erwinia.

Recent intensive studies of $16 \mathrm{~S}$ rDNA sequences suggest that this approach could be used for identification purposes and also to resolve the taxonomic relationships of different species and groups of Erwinia (Kwon et al., 1997; Hauben et al., 1998; Mergaert et al., 1999; Kim et al., 1999). As a result, it has been proposed that the genus Erwinia should be divided into four new genera, namely Erwinia, Pectobacterium, Pantoea and Brenneria. The genus Erwinia has been restricted to six species according to Hauben et al. (1999), E. amylovora, Erwinia mallotivora, Erwinia persicina, Erwinia psidii, Erwinia rhapontici and Erwinia tracheiphila. The resurrected genus Pectobacterium consists of four subspecies of E. carotovora together with E. chrysanthemi, Erwinia cacticida and Erwinia cypripedii. Six species, Erwinia alni, Erwinia nigrifluens, Erwinia paradisiaca, Erwinia quercina, Erwinia rubrifaciens and Erwinia salicis, have been classified in a new genus, Brenneria. Five species previously classified in the genus Erwinia, namely Erwinia ananas, Erwinia herbicola, Erwinia milletiae, Erwinia stewartii and Erwinia uredovora, have been reclassified in the genus Pantoea. This new nomenclature has not generally been accepted by plant pathologists; nevertheless both nomenclatures are currently in use.

Regardless of the taxonomy, it is important to identify bacterial species accurately and rapidly. New approaches based on application of several molecular markers give more information about genome specificity. As well as $16 \mathrm{~S}$ and $23 \mathrm{~S}$ rRNA there are several other candidates: heat-shock proteins (Hsp70, GroEL, Hsp60), the ATPase $\beta$ subunit, RNA polymerases and recombinase A (RecA) can serve as molecular markers for the identification of bacterial pathogens (Ludwig \& Schleifer, 1999). RecA is a multifunctional protein involved in homologous recombination, DNA repair and the SOS response (Eisen, 1995). It is considered to be universally present in prokaryotic and eukaryotic cells as it shows a high degree of sequence conservation. RecA protein and recA gene sequence comparisons have been used to speculate on phylogenetic relationships among genera and species (Lloyd \& Sharp, 1993; Eisen, 1995; Karlin et al., 1995). The recA gene has been used for typing of acinetobacters (Nowak \& Kur, 1995) and for identification of Mycobacterium species (Blackwood et al., 2000) and the Bulkholderia cepacia complex (Mahenthiralingam et al., 2000). Preliminary results showing the usefulness of recA PCR-RFLP for geno- typing of E. carotovora were presented by Waleron et al. (2001).

This paper describes a method based on the analysis of recA gene polymorphism for the identification of the different species and subspecies of the former Erwinia genus. In addition, an analysis of the differentiation between the subspecies of E. carotovora and variation within E. chrysanthemi has been done.

\section{METHODS}

Bacterial strains. One hundred and seventy-seven strains of 19 different species belonging to the former Erwinia genus were tested (Table 1). In addition strains of some plant-pathogenic bacteria [Agrobacterium tumefaciens (3), Pseudomonas savastanoi pv. phaseolicola (4), Xanthomonas vesicatoria (3), Xanthomonas hortorum pv. pelargonii (1)] and of several other bacterial species [Bacillus (now Geobacillus) stearothermophilus (1), Citrobacter freundii (2), Enterobacter (now Pantoea) agglomerans (1), Enterobacter sakazakii (1), Enterobacter cloacae (1), Escherichia coli (4), Klebsiella oxytoca (1), Klebsiella pneumoniae (1), Morganella morganii (1), Proteus vulgaris (1), Salmonella Anantum (1), Salmonella Heidelberg (1), Serratia marcescens (1), 'Shigella shigae' (1), Staphylococcus aureus (1), Staphylococcus epidermidis (1)] were examined.

For DNA preparation, Erwinia, Agrobacterium, Pseudomonas and Xanthomonas strains were grown overnight in tryptic soy broth (TSB; BioMérieux) in shaken culture at $28^{\circ} \mathrm{C}$. E. chrysanthemi and other Enterobacteriaceae were grown at $37^{\circ} \mathrm{C}$ and E. cacticida at $43^{\circ} \mathrm{C}$. Cells were harvested by centrifugation and resuspended in TE buffer $(50 \mathrm{mM}$ Tris/HCl, $40 \mathrm{mM}$ EDTA, $\mathrm{pH} 8 \cdot 0)$. The bacterial DNA was extracted using the SDS/proteinase $\mathrm{K}$ based method described by Ausubel et al. (1992).

Primer design. Oligonucleotide primers were designed on the basis of the sequence of the E. carotovora recA gene described by Zhao \& McEntee (1990). Regions with low variability were chosen. The sequence of each primer (5'-GGTAAAGGGTCTATCATGCG-3' and 5'-CCTTCACCATACATAATTTGGA-3') was checked for homology to other sequences that may also be amplified by them, in the GenBank and EMBL databases using the BLAST N program.

DNA amplification. DNA amplification was performed in $50 \mu \mathrm{l}$ reaction volumes containing $5 \mu \mathrm{l} 10 \times$ reaction buffer (Fermentas), $2.5 \mathrm{mM} \mathrm{MgCl}_{2}, 250 \mu \mathrm{M}$ each of dATP, dCTP, dGTP and dTTP, 20 pmol each primer, $0 \cdot 1 \%$ (v/v) Tween 20, 50-100 ng DNA and $1 \mathrm{U}$ recombinant Taq DNA polymerase (cloned and purified by Dr J. Osipiuk, Department of Microbiology, University of Gdansk). Amplification was performed using a UNOII Biometra thermocycler with initial denaturation $\left(95^{\circ} \mathrm{C}, 3 \mathrm{~min}\right)$, followed by 32 cycles of denaturation $\left(94^{\circ} \mathrm{C}, 1 \mathrm{~min}\right)$, annealing $\left(47^{\circ} \mathrm{C}, 1 \mathrm{~min}\right)$ and extension $\left(72{ }^{\circ} \mathrm{C}, 2 \mathrm{~min}\right)$, with a final extension $\left(72^{\circ} \mathrm{C}, 5 \mathrm{~min}\right)$. The amplified products were electrophoretically separated in $6 \%$ $(\mathrm{w} / \mathrm{v})$ polyacrylamide gel at $120 \mathrm{~V}$ for $10 \mathrm{~h}$ in TBE buffer and visualized with UV light after staining in ethidium bromide $\left(0 \cdot 5 \mu \mathrm{g} \mathrm{ml}^{-1}\right)$.

Restriction fragment length analysis. The amplified DNA fragments were digested with four restriction endonucleases (AluI, HinfI, TasI and Tru1I), which were selected on the basis of the nucleotide sequence of the recA gene of E. carotovora using Vector NTI software. The restriction analysis was performed overnight with $2 \cdot 5 \mathrm{U}$ of each endonuclease using the buffer and temperature recommended by the manufac- 
Table 1. Origin and recA PCR-RFLP groups of the bacterial strains used in this study

\begin{tabular}{|c|c|c|c|}
\hline Bacterial strain* & Host & $\begin{array}{l}\text { Geographic origin and year } \\
\text { of isolation }\end{array}$ & $\begin{array}{l}\text { RFLP } \\
\text { group }\end{array}$ \\
\hline \multicolumn{4}{|c|}{$\begin{array}{l}\text { Erwinia carotovora subsp. atroseptica (Pectobacterium } \\
\text { carotovorum subsp. atrosepticum) }\end{array}$} \\
\hline $16^{\mathrm{a}}$ & Potato & Arizona, USA & 1 \\
\hline $22^{\mathrm{a}}$ & Potato & Arizona, USA & 1 \\
\hline $86^{\mathrm{a}}$ & Potato stem & Peru & 1 \\
\hline $1015^{\mathrm{a}}$ & Soil & Scotland & 1 \\
\hline $147^{\mathrm{k}}$ & & Sweden & 1 \\
\hline $\mathrm{SAC} 12^{\mathrm{e}}$ & & Finland & 1 \\
\hline $185^{\mathrm{d}}$ & & Germany, 1986 & 1 \\
\hline $16 \mathrm{~A}-1^{\mathrm{t}}$ & Potato stem & Poland, 1996 & 1 \\
\hline $17 \mathrm{~A}-1^{\mathrm{t}}$ & Potato stem & Poland, 1996 & 1 \\
\hline $7 \mathrm{~A}-1^{\mathrm{t}}$ & Potato stem & Poland, 1996 & 1 \\
\hline $1043^{\mathrm{a}}$ & Potato stem & Scotland & 2 \\
\hline $324^{b}$ & Potato & Greece, 1971 & 2 \\
\hline $75 \mathrm{~B}-1^{\mathrm{t}}$ & Potato tuber & Poland, 1996 & 2 \\
\hline \multicolumn{4}{|c|}{$\begin{array}{l}\text { Erwinia carotovora subsp. carotovora (Pectobacterium } \\
\text { carotovorum subsp. carotovorum) }\end{array}$} \\
\hline ATCC $15713^{\mathrm{Ti}}$ & Potato & Denmark & 3 \\
\hline $\mathrm{PH} 200^{\mathrm{e}}$ & Potato stem & Finland & 3 \\
\hline $103^{\mathrm{a}}$ & Potato tuber & Scotland & 3 \\
\hline $140^{\mathrm{a}}$ & Potato & Arizona, USA & 3 \\
\hline $142^{\mathrm{a}}$ & Potato & Arizona, USA & 3 \\
\hline $1 \mathrm{~A}-1^{\mathrm{t}}$ & Potato stem & Poland, 1996 & 3 \\
\hline $15 \mathrm{~A}-3^{\mathrm{t}}$ & Potato stem & Poland, 1996 & 3 \\
\hline $16 \mathrm{~A}-2^{\mathrm{t}}$ & Potato stem & Poland, 1996 & 3 \\
\hline NAP $120 / 408^{j}$ & Sunflower & Hungary, 1986 & 4 \\
\hline $116^{\mathrm{a}}$ & Swede & Scotland, 1977 & 4 \\
\hline $142^{\mathrm{a}}$ & Potato & Arizona, USA & 4 \\
\hline $156^{\mathrm{a}}$ & Potato stem & Tasmania, 1973 & 4 \\
\hline $536 / 96^{\mathrm{f}}$ & Cabbage & Poland, 1996 & 4 \\
\hline $539 / 96^{\mathrm{f}}$ & Leek & Poland, 1996 & 4 \\
\hline $545 / 96^{\mathrm{f}}$ & Celery & Poland, 1996 & 4 \\
\hline $548 / 96^{\mathrm{f}}$ & Carrot & Poland, 1996 & 4 \\
\hline $\mathrm{AH} 2^{\mathrm{g}}$ & & Oregon, USA & 4 \\
\hline $135^{\mathrm{n}}$ & Potato & Germany & 4 \\
\hline $56 \mathrm{~A}-15^{\mathrm{t}}$ & Potato stem & Poland, 1996 & 4 \\
\hline $95^{\mathrm{n}}$ & Cabbage & Germany & 5 \\
\hline $137^{\mathrm{a}}$ & Potato & Arizona, USA & 5 \\
\hline $143^{\mathrm{a}}$ & Potato & Tasmania & 5 \\
\hline $793^{\mathrm{b}}$ & Hyacinth tuber & Greece, 1981 & 5 \\
\hline LA $128^{\mathrm{c}}(7)$ & & USA & 5 \\
\hline JA $024 / 319^{j}$ & Hyacinth tuber & Hungary, 1986 & 5 \\
\hline $8982^{1}$ & & Ukraine & 5 \\
\hline $23 \mathrm{~A}-4^{\mathrm{t}}$ & Potato stem & Poland, 1996 & 5 \\
\hline $66 \mathrm{~A}-1^{\mathrm{t}}$ & Potato stem & Poland, 1996 & 5 \\
\hline $160^{\mathrm{a}}$ & Potato stem & Tasmania, 1970 & 6 \\
\hline $161^{\mathrm{a}}$ & Potato stem & Tasmania, 1970 & 6 \\
\hline $248^{\mathrm{a}}$ & Soil water & Scotland, 1982 & 6 \\
\hline $171^{\mathrm{a}}$ & Potato & Tasmania, 1970 & 7 \\
\hline $180^{\mathrm{a}}$ & Potato tuber & Peru, 1979 & 7 \\
\hline $174^{\mathrm{a}}$ & Potato tuber & Peru, 1977 & 8 \\
\hline $791^{\mathrm{b}}$ & Cabbage & Greece, 1981 & 8 \\
\hline $332^{\mathrm{k}}$ & & Sweden & 8 \\
\hline $176^{\mathrm{a}}$ & Potato stem & Peru, 1977 & 9 \\
\hline $178^{\mathrm{a}}$ & Potato stem & Peru, 1978 & 10 \\
\hline
\end{tabular}


Table 1 (cont.)

\begin{tabular}{|c|c|c|c|}
\hline Bacterial strain* & Host & $\begin{array}{l}\text { Geographic origin and year } \\
\text { of isolation }\end{array}$ & $\begin{array}{l}\text { RFLP } \\
\text { group }\end{array}$ \\
\hline $179^{\mathrm{a}}$ & Potato root & Peru, 1979 & 11 \\
\hline $249^{\mathrm{a}}$ & Potato tuber & Scotland, 1982 & 12 \\
\hline $582^{\mathrm{d}}$ & Chicory & Switzerland, 1985 & 13 \\
\hline $\mathrm{MN} 12-2^{\mathrm{t}}$ & Parsley & Poland, 1999 & 13 \\
\hline $\mathrm{MN} \mathrm{6-1}$ & Carrot & Poland, 1999 & 13 \\
\hline $\mathrm{MN} \mathrm{6-2^{ \textrm {t } }}$ & Carrot & Poland, 1999 & 13 \\
\hline $\operatorname{Re}-4^{t}$ & Celery & Poland, 1999 & 13 \\
\hline $\operatorname{Re}-5^{t}$ & Carrot & Poland, 1999 & 13 \\
\hline $\mathrm{MN} 10-1^{\mathrm{t}}$ & Onion & Poland, 1999 & 13 \\
\hline $134 \mathrm{~A}-2^{\mathrm{t}}$ & Potato stem & Poland, 1996 & 14 \\
\hline $537 / 96^{\mathrm{t}}$ & Cabbage & Poland, 1999 & 15 \\
\hline $159^{\mathrm{b}}$ & Cyclamen & Greece, 1967 & 16 \\
\hline $333^{\mathrm{b}}$ & Artichoke & Greece, 1971 & 17 \\
\hline $573^{\mathrm{d}}$ & Cabbage & Switzerland, 1983 & 17 \\
\hline JL 1105 (SR 319) $^{\mathrm{c}}$ & Soil & & 18 \\
\hline $43 \mathrm{~B}-1^{\mathrm{t}}$ & Potato tuber & Poland, 1996 & 18 \\
\hline LMG $2404^{\mathrm{e}}$ & Potato & Denmark & 19 \\
\hline $143 \mathrm{~A}-1^{\mathrm{t}}$ & Potato stem & Poland, 1997 & 20 \\
\hline $143 \mathrm{~B}-1^{\mathrm{t}}$ & Potato tuber & Poland, 1997 & 20 \\
\hline \multicolumn{4}{|l|}{$\begin{array}{l}\text { Erwinia carotovora subsp. betavasculorum (Pectobacterium } \\
\text { carotovorum subsp. betavasculorum) }\end{array}$} \\
\hline $\begin{array}{l}\text { ATCC 43762 } \\
\text { NCPPB } 2795)\end{array}$ & Sugar beet & California, USA, 1972 & 21 \\
\hline LA $129(=\mathrm{Ecb} 168)^{\circ}$ & Sugar beet & USA & 21 \\
\hline CFBP $2121^{\mathrm{u}}$ ( = LMG 2463) & Sugar beet & USA, 1974 & 21 \\
\hline CFBP $1520^{u}$ & Sunflower & Mexico & 21 \\
\hline SF142, $2^{\mathrm{u}}$ & Artichoke & La Reunion, 1986 & 21 \\
\hline CU $0307^{p}$ & Sugar beet & Washington, USA & 21 \\
\hline CU $0341^{p}$ ( = LMG 2462, NCPPB 2793) & Sugar beet & California, USA, 1974 & 21 \\
\hline CU $0342^{\mathrm{p}}(=\mathrm{NCPPB} 3075)$ & Sugar beet & California, USA & 21 \\
\hline CU $0350^{p}$ & Sugar beet & Arizona, USA & 21 \\
\hline \multicolumn{4}{|l|}{$\begin{array}{l}\text { Erwinia carotovora subsp. odorifera (Pectobacterium } \\
\text { carotovorum subsp. odoriferum) }\end{array}$} \\
\hline CFBP $1878^{\mathrm{u}}\left(=\mathrm{LMG} 17566^{\mathrm{T}}\right.$, NCPPB 3839) & Celery & France, 1979 & 22 \\
\hline $482^{\mathrm{a}}(=\mathrm{NCPPB} 3840)$ & Witloof chicory & France & 22 \\
\hline $487^{\mathrm{a}}(=353)$ & Witloof chicory & France & 22 \\
\hline CFBP $1880^{u}$ & Witloof chicory & France, 1979 & 22 \\
\hline CFBP $1959^{u}$ & Witloof chicory & France, 1980 & 22 \\
\hline CFBP $1893^{u}$ & Celery & France, 1976 & 22 \\
\hline CFBP $3260^{\mathrm{u}}(=1654,1)^{\mathrm{u}}$ & Leek & France, 1980 & 22 \\
\hline CFBP $3259^{\mathrm{u}}(=1646,2)^{\mathrm{u}}$ & Leek & France, 1980 & 22 \\
\hline CFBP $1879^{u}$ & Celery & France, 1979 & 22 \\
\hline CFBP $1892^{u}$ & Celery & France, 1981 & 22 \\
\hline CFBP $2154^{u}$ & Celery & France, 1982 & 22 \\
\hline CFBP $2155^{u}$ & Celery & France, 1983 & 22 \\
\hline \multicolumn{4}{|l|}{$\begin{array}{l}\text { Erwinia carotovora subsp. wasabiae (Pectobacterium } \\
\text { carotovorum subsp. wasabiae) }\end{array}$} \\
\hline ATCC $43316^{\mathrm{Ti}}\left(=481^{\mathrm{a}}, \mathrm{CFBP} 3304^{\mathrm{u}}, \mathrm{NCPPB} 370\right.$, SR 91) & Horseradish & Japan & 23 \\
\hline $917^{\mathrm{a}}(=\mathrm{NCPPB} 3702)$ & Horseradish & Japan & 23 \\
\hline $918^{\mathrm{a}}(=\mathrm{NCPPB} 3703)$ & Horseradish & Japan & 23 \\
\hline $919^{\mathrm{a}}($ = NCPPB 3704) & Horseradish & Japan & 23 \\
\hline CFBP $3308^{u}$ ( = ICMP 9125) & Horseradish & Japan, 1985 & 23 \\
\hline
\end{tabular}


Table 1 (cont.)

\begin{tabular}{|c|c|c|c|}
\hline Bacterial strain* & Host & $\begin{array}{c}\text { Geographic origin and year } \\
\text { of isolation }\end{array}$ & $\begin{array}{l}\text { RFLP } \\
\text { group }\end{array}$ \\
\hline \multicolumn{4}{|l|}{ Erwinia chrysanthemi (Pectobacterium chrysanthemi) } \\
\hline $\operatorname{ET~} 3^{\mathrm{y}}$ & Tomato & Martinique, 1987 & 33 \\
\hline $1240^{\mathrm{y}}$ & Carnation & Denmark, 1956 & 33 \\
\hline $0600^{\mathrm{a}}$ & Chrysanthemum & Holland & 33 \\
\hline $0174^{\mathrm{a}}$ & Saintpaulia & Germany & 34 \\
\hline $1260^{\mathrm{a}}$ & Potato & Germany & 34 \\
\hline $4061^{\mathrm{a}}$ & Colocasia & Solomon Islands & 34 \\
\hline $564^{\mathrm{b}}$ & Carnation & Greece, 1976 & 35 \\
\hline $0872^{\mathrm{z}}(=\mathrm{PD} 484)$ & Potato & Holland & 35 \\
\hline $1086^{\mathrm{z}}$ & Potato & Finland & 35 \\
\hline $1076^{\mathrm{z}}$ & Potato & Spain & 35 \\
\hline $877^{\mathrm{z}}$ & Potato & Holland & 35 \\
\hline $1306^{\mathrm{z}}$ & Potato & Spain & 35 \\
\hline $9^{d}$ & & USA & 36 \\
\hline $260^{\mathrm{d}}$ & & USA & 36 \\
\hline $4069^{\mathrm{a}}(=\mathrm{B} 374)$ & Pelargonium & Comoro Island & 37 \\
\hline ATCC $11663^{\mathrm{Ti}}(=\mathrm{NCPPB} 402)$ & Chrysanthemum & USA, 1956 & 38 \\
\hline $3262^{y}$ & Chicory & France, 1981 & 38 \\
\hline $1891^{\mathrm{y}}$ & Tobacco & USA & 39 \\
\hline $946^{\mathrm{z}}$ & Potato & Holland & 40 \\
\hline $996^{\mathrm{z}}$ & Potato & Holland & 41 \\
\hline $4078^{\mathrm{a}}(=1271)$ & Corn & Egypt, 1961 & 42 \\
\hline SF109-1 $1^{y}$ & Sunflower & France, 1986 & 43 \\
\hline $\mathrm{SH} 230^{\mathrm{y}}(=\mathrm{C} 413)$ & Tomato & Cuba & 44 \\
\hline $998^{\mathrm{z}}$ & Potato & Holland & 45 \\
\hline $4062^{a}$ & Agalonema & St Lucia & 46 \\
\hline $0597^{\mathrm{z}}$ & Potato & Peru & 47 \\
\hline \multicolumn{4}{|l|}{ Erwinia cacticida (Pectobacterium cacticida) } \\
\hline 72-1"w ( = ICMP 7452-81, ICPB EC188) & Opuntia & Arizona, USA, 1958 & 29 \\
\hline CFBP $3628^{\mathrm{Td}}(=$ ATCC 49489$)$ & Carnegiena gigantea & Arizona, USA, 1958 & 30 \\
\hline DU89-8, $1^{\mathrm{w}}(=$ ATCC 49483$)$ & Opuntia & Australia, 1989 & 30 \\
\hline $66-50^{\mathrm{w}}(=\mathrm{ICPB}$ EC290) & Carnegiena gigantea & Arizona, USA, 1966 & 30 \\
\hline Texas $29^{\mathrm{w}}$ & Opuntia & Texas, USA, 1971 & 30 \\
\hline Texas28w ( = ICPB 296) & Opuntia & Texas, USA, 1971 & 30 \\
\hline $78-28^{\mathrm{w}}(=\mathrm{ATCC} 49482, \mathrm{ICPB}$ EC283) & $\begin{array}{r}\text { Stenocereus } \\
\text { gummosus }\end{array}$ & Mexico, 1978 & 30 \\
\hline $106^{\mathrm{w}}(=\mathrm{ICPB}$ EC189) & Carnegiena gigantea & Arizona, USA, 1958 & 30 \\
\hline NCPPB $671^{\mathrm{w}}$ ( = Dye EH4) & Carnegiena gigantea & Arizona, USA, 1980 & 30 \\
\hline DU $89-5,1^{\mathrm{w}}$ & Opuntia & Australia, 1989 & 31 \\
\hline DU $89-7,3^{w}$ & Opuntia & Australia, 1989 & 31 \\
\hline $66-19-1^{w}$ & Opuntia & Arizona, USA, 1966 & 31 \\
\hline $62-63^{\mathrm{w}}(=\mathrm{ATCC} 49485)$ & Opuntia & Arizona, USA, 1962 & 31 \\
\hline $623-2^{\mathrm{w}}$ & Carnegiena gigantea & Arizona, USA, 1959 & 31 \\
\hline $88-6^{\mathrm{w}}$ & Carnegiena gigantea & Arizona, USA, 1988 & 31 \\
\hline \multicolumn{4}{|l|}{ Erwinia cypripedii (Pectobacterium cypripedii) } \\
\hline $\begin{array}{l}\text { ATCC } 29267^{\mathrm{Ti}}\left(=\mathrm{CU} 5464^{\mathrm{p}}, \mathrm{NCPPB} 3004, \mathrm{CFBP} 3613^{\mathrm{d}} \text {, }\right. \\
\text { PDDCC 0695) }\end{array}$ & Orchid & California, USA, 1950 & 24 \\
\hline CU $5465^{p}$ & Orchid & California, USA, 1950 & 24 \\
\hline $440^{\mathrm{a}}(=\mathrm{NCPPB} 752)$ & Orchid & California, USA, 1950 & 24 \\
\hline \multicolumn{4}{|l|}{ Erwinia amylovora } \\
\hline ATCC $15580^{\mathrm{Ti}}\left(=\mathrm{LMG} 2024^{\mathrm{e}}\right)$ & Pear & UK & 32 \\
\hline $394^{x}$ & Pear & Poland & 32 \\
\hline $661^{x}$ & Rowan & Poland & 32 \\
\hline $691^{x}$ & Apple & Poland & 32 \\
\hline
\end{tabular}


Table 1 (cont.)

\begin{tabular}{|c|c|c|c|}
\hline Bacterial strain* & Host & $\begin{array}{l}\text { Geographic origin and year } \\
\text { of isolation }\end{array}$ & $\begin{array}{l}\text { RFLP } \\
\text { group }\end{array}$ \\
\hline $1056^{\mathrm{b}}(=$ BPIC 1989) & Quince & Greece, 1987 & 32 \\
\hline $1628^{b}(=$ BI 1990) & Quince & Bulgaria, 1990 & 32 \\
\hline $\mathrm{G}-7^{\mathrm{s}}$ & Pear or apple & Canada & 32 \\
\hline E $7001 M^{s}$ & Mountain ash & Canada & 32 \\
\hline $\mathrm{G}-5^{\mathrm{s}}$ & Pear or apple & Canada & 32 \\
\hline \multicolumn{4}{|l|}{ Erwinia persicina } \\
\hline ATCC $35998^{\mathrm{Ti}}\left(=\mathrm{LMG} 11254\right.$, CFBP $3622^{\mathrm{d}}$ ) & Tomato & Japan & 49 \\
\hline $\operatorname{ATCC~} 756^{\circ}$ & Human & Texas, USA & 49 \\
\hline \multicolumn{4}{|l|}{ Erwinia rhapontici } \\
\hline $421^{\mathrm{a}}(=\mathrm{NCPPB} 139)$ & Rhubarb & UK, 1940 & 21 \\
\hline $422^{\mathrm{a}}(=$ ATCC 29284, LMG 3292, NCPPB 1739) & Rhubarb & UK, 1965 & 21 \\
\hline $\begin{array}{l}\text { ATCC } 29283^{\mathrm{Ti}}\left(=\mathrm{CFBP} 3618^{\mathrm{d}}, \text { NCPPB } 1578^{\mathrm{e}}, 4240^{\mathrm{p}}, \mathrm{LMG}\right. \\
2688)\end{array}$ & Rhubarb & UK, 1963 & 53 \\
\hline \multicolumn{4}{|l|}{ Erwinia mallotivora } \\
\hline CFBP 2503u ( = ATCC 29573T, LMG 2708) & Mallotus japonicus & Japan, 1975 & 55 \\
\hline \multicolumn{4}{|l|}{ Erwinia psidii } \\
\hline CFBP $3627^{\mathrm{u}}\left(=\right.$ ATCC $\left.49406^{\mathrm{T}}, \mathrm{NCPPB} 3555\right)$ & Common guava & Brazil, 1982 & 54 \\
\hline \multicolumn{4}{|l|}{ Erwinia tracheiphila } \\
\hline CBP $2355^{\mathrm{u}}\left(=\operatorname{ATCC} 33245^{\mathrm{T}}\right)$ & Cucumber & USA, 1972 & 57 \\
\hline \multicolumn{4}{|l|}{ Erwinia ananas (Pantoea ananatis) } \\
\hline ATCC $33244^{\mathrm{Ti}}\left(=485^{\mathrm{a}}, \mathrm{LMG} 2665, \mathrm{NCPPB} 1846\right)$ & Pineapple & Brazil, 1965 & 25 \\
\hline $\mathrm{TM}^{\mathrm{r}}$ & Mulberry leaves & Japan, 1993 & 25 \\
\hline Mei $^{r}$ & Mulberry pyralid & Japan, 1993 & 25 \\
\hline INE14 ${ }^{r}$ & Rice & Japan, 1991 & 26 \\
\hline \multicolumn{4}{|l|}{ Erwinia herbicola (Pantoea agglomerans) } \\
\hline Mei3 $3^{r}$ & Mulberry pyralid & Japan, 1991 & 27 \\
\hline $\mathrm{DW} 1^{\mathrm{r}}$ & Mulberry leaves & Japan, 1991 & 27 \\
\hline ATCC $33243^{\mathrm{Ti}}(=\mathrm{LMG} 2565)$ & Cereals & Canada & 28 \\
\hline $\mathrm{BME}^{\mathrm{r}}$ & Silkworm & Japan, 1994 & 28 \\
\hline MBE$^{r}$ & Mulberry leaves & Japan, 1994 & 28 \\
\hline $\mathrm{H} 1^{\mathrm{g}}$ & & & 28 \\
\hline \multicolumn{4}{|l|}{ Erwinia milletiae (Pantoea agglomerans) } \\
\hline ATCC $33261^{\mathrm{Ti}}(=$ NCPPB 2519) & Wisteria floribunda & Japan & 28 \\
\hline $\begin{array}{l}\text { Pantoea sp. } \\
\text { MW } 1^{\mathrm{g}}\end{array}$ & \multicolumn{2}{|c|}{ Pantoea sp. } & 28 \\
\hline \multicolumn{4}{|l|}{ Erwinia stewartii (Pantoea stewartii) } \\
\hline ATCC $8199^{\mathrm{Ti}}$ & Corn & USA & 48 \\
\hline ST $1^{\mathrm{g}}$ & & Ohio, USA, 1967 & 48 \\
\hline \multicolumn{4}{|l|}{ Erwinia uredovora (Pantoea ananatis) } \\
\hline ATCC $19321^{\mathrm{Ti}}(=\mathrm{LMG} 2676)$ & Wheat & USA, 1954 & 25 \\
\hline \multicolumn{4}{|l|}{ Erwinia nigrifluens (Brenneria nigrifluens) } \\
\hline $\begin{array}{l}\text { ATCC } 13028^{\mathrm{Ti}}\left(=1709^{\mathrm{b}}, \mathrm{CFBP} 3616^{\mathrm{d}}, \mathrm{LMG} 2694,\right. \\
\text { NCPPB564) }\end{array}$ & Walnut & USA, 1955 & 51 \\
\hline \multicolumn{4}{|l|}{ Erwinia quercina (Brenneria quercina) } \\
\hline CFBP $367^{\mathrm{i}}\left(=\right.$ ATCC $29281^{\mathrm{T}}$, NCPPB 1852$)$ & Oak & USA, 1964 & 56 \\
\hline
\end{tabular}


Table 1 (cont.)

\begin{tabular}{|c|c|c|c|}
\hline Bacterial strain* & Host & $\begin{array}{l}\text { Geographic origin and year } \\
\text { of isolation }\end{array}$ & $\begin{array}{l}\text { RFLP } \\
\text { group }\end{array}$ \\
\hline $\begin{array}{l}\text { Erwinia rubrifaciens (Brenneria rubrifaciens) } \\
\text { ATCC } 29291^{\mathrm{Ti}}\left(=710^{\mathrm{b}}, \text { CFBP } 3619^{\mathrm{d}}, \text { LMG } 2709, \mathrm{NCPPB}\right. \\
2020)\end{array}$ & Walnut & USA, 1963 & 50 \\
\hline $\begin{array}{l}\text { Erwinia salicis (Brenneria salicis) } \\
\text { ATCC } 15712^{\mathrm{Ti}}\left(=\text { CFBP } 802^{\mathrm{d}}, \text { LMG 2698) }\right.\end{array}$ & Willow & UK, 1957 & 52 \\
\hline $\begin{array}{l}\text { Names of organisms in parentheses are those proposed by H } \\
\text { Dundee, UK; b, P. G. Psallidas, Benaki Phytopathological In } \\
\text { Corvallis, OR, USA; d, K. Geider, Max-Planck-Institut für } \\
\text { Helsinki, Finland; f, M. Guranowska, Collection of Plant Pa } \\
\text { Ohio State University, Columbus, OH, USA; i, S.-W. Kwon } \\
\text { Korea; j, J. Németh, Plant Health and Soil Conservation, Sta } \\
\text { of Agricultural Sciences, Uppsala, Sweden; 1, B. Sharga, Uzhgo } \\
\text { Research on Cultivated Plants, Groß Lüsewitz, Germany; n, } \\
\text { Germany; o, C. M. O'Hara, Centers for Disease Control and I } \\
\text { USA; r, K. Watanabe, National Institute of Sericulture and En } \\
\text { Agriculture \& Agri-Food Canada, Southern Crop Protection } \\
\text { Biotechnology, Intercollegiate Faculty of Biotechnology, Gda } \\
\text { de Recherches Agronomique d'Angers, Angers, France; w, T. } \\
\text { of Pomology, Skierniewice, Poland; y, Y. Bertheau, Institut N } \\
\text { Plant Research International, Wageningen, The Netherlands. }\end{array}$ & $\begin{array}{l}\text { al. (1998). a } \\
\text { ifissia, Ath } \\
\text { gie, Rosenh } \\
\text { f the Instit } \\
\text { al Institute } \\
\text { County Bar } \\
\text { University, } \\
\text { tigall, Fede } \\
\text { n, Atlanta, } \\
\text { cal Science, } \\
\text { Research C } \\
\text { nd; u, L. G } \\
\text { iiversity of } \\
\text { e la Recher }\end{array}$ & $\begin{array}{l}\text { ctish Crop Research Institute, } \\
\text { c, V. Stockwell, Oregon State } \\
\text {; e, H. Saarilahti, University } \\
\text { Protection, Poznan, Poland; g } \\
\text { ral Science \& Technology, RI } \\
\text { Hungary; k, P. Person, Swedisl } \\
\text { Ch. Wegener, Federal Centre } \\
\text { r Breeding Research on Cultiv } \\
\text { S. V. Beer, Cornell University, } \\
\text { araki, Japan; s, D. M. Hunter, } \\
\text { da; t, Department of Plant Prc } \\
\text { ute de la Recherche Agronomi } \\
\text { scon, AZ, USA; x, P. Sobiczews } \\
\text { iique, Paris, France; z, J. M. va }\end{array}$ & $\begin{array}{l}\text { niversity, } \\
\text { Helsinki, } \\
\text {. Coplin, } \\
\text {, Suwon, } \\
\text { University } \\
\text { Breeding } \\
\text { ed Plants, } \\
\text { laca, NY, } \\
\text { ree Fruits } \\
\text { ction and } \\
\text { e, Centre } \\
\text { Institute } \\
\text { der Wolf, }\end{array}$ \\
\hline
\end{tabular}

turers (Fermentas). Restriction fragments were separated in a $12 \%(\mathrm{w} / \mathrm{v})$ polyacrylamide gel at $120 \mathrm{~V}$ for $10 \mathrm{~h}$ in TBE buffer and visualized with UV light after staining in ethidium bromide $\left(0 \cdot 5 \mu \mathrm{g} \mathrm{ml}^{-1}\right)$.

\section{RESULTS}

DNA isolated from the cells of the bacteria of 19 former Erwinia species and five subspecies of E. carotovora, three other genera of plant-pathogenic bacteria (Agrobacterium tumefaciens, Pseudomonas savastano $i \mathrm{pv}$. phaseolicola, Xanthomonas spp.) and 16 other species of bacteria, mainly from the family Enterobacteriaceae, were used as a target in PCR reactions with primers designed to be complementary to the E. carotovora rec $A$ gene. The primers generated amplification products of approximately $730 \mathrm{bp}$ with target DNA isolated for all of the Erwinia strains. At least one PCR product for each species and subspecies was sequenced directly in both directions and the presence of the specific restriction sites was confirmed (M. Waleron, K. Waleron \& E. Łojkowska, unpublished results).

PCR products were digested by four restriction endonucleases (AluI, HinfI, TasI and Tru1I) in four separate reactions (Fig. 1). The smallest differentiation of RFLP patterns was observed with $\operatorname{HinfI}$, which gave only seven different patterns with all Erwinia strains (Fig. 1a). The greatest differentiation was obtained after digestion of the PCR product with TasI, which gave 31 patterns (Fig. 1b). AluI and Tru1I gave 10 and 14 RFLP patterns, respectively (Fig. 1c, d). The results of the recA RFLP analysis of PCR product based on the number of bands and their position revealed the presence of 57 different recA combined RFLP patterns (restriction groups) (Table 2). The combined patterns of the restriction analysis of the $\operatorname{rec} A$ gene were consistently different and characteristic for most of the species and subspecies tested (Table 1).

No amplification products were obtained from plantpathogenic bacteria other than Erwinia. Of the other bacteria, only the DNA isolated from cells of bacteria belonging to Enterobacteriaceae (Shigella spp., Serratia spp. and Klebsiella spp.) yielded a product of similar size to that amplified from Erwinia species. However, restriction patterns were clearly different to those obtained for Erwinia species (data not shown).

Strains belonging to four species (E. amylovora, E. cypripedii, E. persicina and E. stewartii) and three subspecies of E. carotovora (subsp. betavasculorum, odorifera and wasabiae) occupied single RFLP groups based on recA PCR-RFLP (Table 1). In many other cases only one strain of a species was tested and examination of additional strains may reveal different RFLP patterns. Copies of the same strain obtained from different laboratories were tested and in all cases these showed the same RFLP profile (Table 1 ). Strains of E. ananas, E. carotovora subsp. atroseptica, E. herbicola and E. rhapontici occupied two different RFLP groups and those of E. cacticida three groups (Table 1). The highest diversity of the $\operatorname{rec} A$ gene was observed among 57 strains of E. carotovora subsp. carotovora (18 groups) and 26 strains of E. chrysanthemi (15 groups) (Tables 1 and 2).

\section{DISCUSSION}

One hundred and seventy-seven strains of Erwinia, comprising 19 different species, were tested using PCRRFLP with primers from the recA gene sequence. Fifty- 

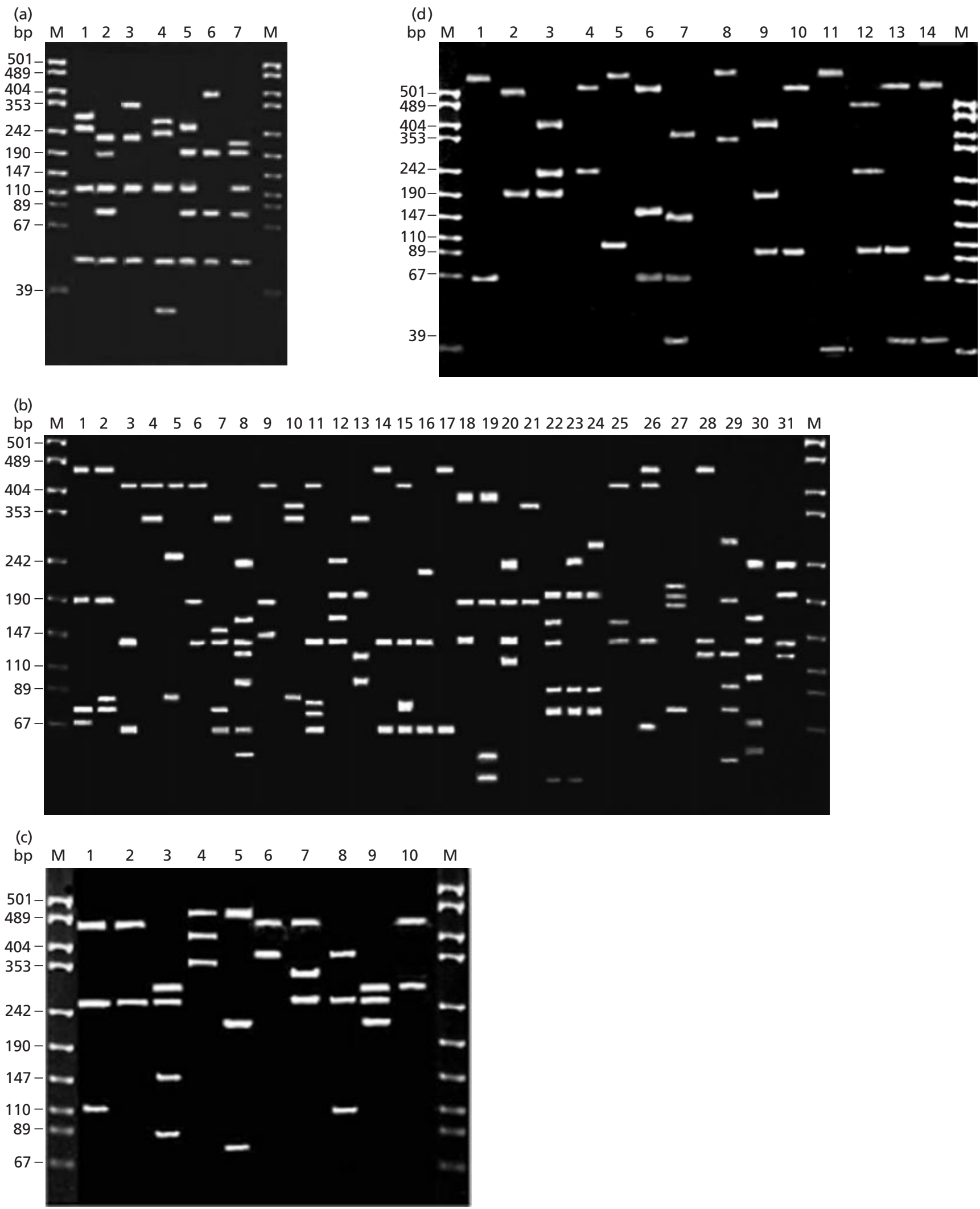

Fig. 1. Restriction analysis of a recA gene fragment amplified by PCR. RFLP patterns were obtained after digestion of $P C R$ products with Hinfl (a), Tasl (b), Alul (c) and Tru1l (d). Lane M, molecular mass size markers (pUC18/Mspl); other lanes, digestion products of recA gene fragments amplified from the genomic DNA of Erwinia. Lane numbers correspond to the PCR-RFLP patterns obtained for each endonuclease as shown in Table 2. 
seven different RFLP groups were identified. Analysis of the results of the recA PCR-RFLP genotyping of 140 strains of pectinolytic Erwinia, reclassified by Hauben et al. (1998) on the basis of $16 \mathrm{~S}$ rDNA sequence into the resurrected genus Pectobacterium, indicated the presence of 42 different RFLP groups (combined restriction profiles). The highest number of restriction profiles was obtained when recA gene fragments of E. carotovora subsp. carotovora and E. chrysanthemi were analysed (Table 1). A similar high level of variability in these species was observed in serological studies (De Boer \& McNaughton, 1987; Samson et al., 1989) and in RFLP analysis of the sequences of genes encoding isoenzymes from the pectate lyase family (Darrasse et al., 1994; Nassar et al., 1996).

In contrast, the 13 strains from E. carotovora subsp. atroseptica belong to only two specific RFLP groups, 1 and 2 (Table 1 ). The low variability in this subspecies is in agreement with serological studies that indicated the presence of only a few serogroups (De Boer \& McNaughton, 1987). Similarly, only two RFLP groups were obtained after analysis of the polymorphism of the genes encoding pectate lyase (Darrasse et al., 1994; Śledź et al., 2000). A low level of variation was also observed in PCR for enterobacterial repetitive intergenic consensus (ERIC) sequences (Toth et al., 1999b).

Four other pectolytic Erwinia species, E. cypripedii, E. carotovora subsp. betavasculorum, E. carotovora subsp. odorifera and E. carotovora subsp. wasabiae, show only one specific combined RFLP profile each, whereas the 15 strains of E. cacticida give three different patterns (Table 1). This is in accordance with results reported by Alcorn et al. (1991) who described differences between strains of E. cacticida based on their physiology, pathogenicity and DNA homology.

The non-pectinolytic Erwinia strains, E. amylovora and E. persicina, gave one specific restriction pattern for each species, which suggests a low degree of diversity (Table 1). Although the strains of E. amylovora used were isolated from different Rosaceae plants growing in different countries, they nevertheless belonged to the same recA PCR-RFLP group (Table 1). The low variability among $E$. amylovora strains has also been observed in biochemical (Dye, 1968; Verdonck et al., 1987), serological (Vantomme et al., 1982), DNA hybridization (Brenner et al., 1974) and PFGE studies (Zhang \& Geider, 1997).

For E. rhapontici two RFLP groups were observed. Two strains were assigned to group 21 and were identical to strains of E. carotovora subsp. betavasculorum; a single strain was in the unique group 53 (Table 1). Verdonck et al. (1987) reported that E. rhapontici strains form three groups. One of them was common for E. rhapontici and E. carotovora strains. However, in biochemical and DNA hybridization tests, the type strain of E. rhapontici was very similar to the strains of E. persicina (Hao et al., 1990). Phylogenetic analyses of Erwinia species based on $16 \mathrm{~S}$ rDNA sequences also confirm these observations (Kwon et al., 1997; Hauben et al., 1998).
Analysis of the recA gene in species which have been reclassified in the genus Pantoea (E. ananas, E. herbicola, E. milletiae, E. stewartii and E. uredovora) indicated the presence of five RFLP patterns, some common to more than one species (Table 1). Only E. stewartii strains belonged to one RFLP group. E. ananas strains belonged to two different RFLP groups, 25 and 26, but group 25 also included a single strain of E. uredovora (Table 1). This is in agreement with the results of DNA-DNA hybridization (Mergaert et al., 1993) and analysis of $16 \mathrm{~S}$ rDNA (Kwon et al., 1997; Kim et al., 1999), indicating a high degree of similarity between the two species. On that basis, these two pathogens have been described as being different pathovars of the new species Pantoea ananas, pv. ananas and pv. uredovora. It is interesting to note that strains of E. ananas and E. uredovora that have been reported to be ice-nucleation-active (Watanabe \& Sato, 1998) were placed into RFLP group 25, whereas the strains without this activity were in RFLP group 26.

Six strains of E. herbicola belong to two different RFLP groups, 27 and 28, one of which, group 28, also includes single strains of E. milletiae and Pantoea sp. (Table 1). This is in agreement with data from molecular tests (Brenner et al., 1984; Lind \& Ursing, 1986; Beji et al., 1988) and phenotypic data (Mergaert et al., 1984; Verdonck et al., 1987), which suggest that E. herbicola and E. milletiae are closely related; even their names are synonymous and they have both been reclassified in the genus Pantoea (Gavini et al., 1989).

Strains of E. nigrifluens, E. quercina, E. rubrifaciens and E. salicis, which have been reclassified into the genus Brenneria, each gave one distinct pattern (Table 1). Numerical taxonomic analysis (Verdonck et al., 1987) and $16 \mathrm{~S}$ rDNA sequence analysis (Hauben et al., 1998) of these species showed that they form separate homogeneous groups and are distinct from each other.

The degree of genetic diversity in the different species indicated by the number of RFLP groups obtained per species could be tentatively associated to host specificity and host range of the bacteria, and to their geographical, spatial and temporal distributions. Species exhibiting host specificity and a narrow host range in a defined climate, such as E. amylovora and E. carotovora subsp. atroseptica, infecting rosaceous plants and potato, respectively, would tend to be genetically homogeneous. There was probably less evolutionary pressure to adapt and diversify than in the case of species with a wide host and geographical range, such as E. chrysanthemi and E. carotovora subsp. carotovora (Perombelon \& Kelman, 1980), both with a large number of recA RFLP groups.

Nassar et al. (1996) showed in PCR-RFLP analysis of the genes encoding pectate lyases that at least some of their groupings could be associated with hosts and geographical regions. However, no correlation could be 
Table 2. RFLP groups obtained on the basis of restriction analysis of recA gene fragments with four restriction endonucleases

\begin{tabular}{|c|c|c|c|c|c|c|}
\hline \multirow[t]{2}{*}{$\begin{array}{l}\text { RFLP } \\
\text { group* }\end{array}$} & \multicolumn{4}{|c|}{$\begin{array}{c}\text { PCR-RFLP patterns for specific restriction } \\
\text { endonucleases } \dagger\end{array}$} & \multirow[t]{2}{*}{ Erwinia species assigned } & \multirow[t]{2}{*}{$\begin{array}{l}\text { No. of } \\
\text { strains }\end{array}$} \\
\hline & TasI & $\operatorname{Tru} 1 \mathrm{I}$ & $A l u \mathrm{I}$ & HinfI & & \\
\hline 1 & 1 & 1 & 1 & 1 & E. carotovora subsp. atroseptica & 10 \\
\hline 2 & 2 & 1 & 1 & 1 & & 3 \\
\hline 3 & 3 & 6 & 6 & 3 & E. carotovora subsp. carotovora & 8 \\
\hline 4 & 5 & 2 & 2 & 2 & & 11 \\
\hline 5 & 4 & 2 & 2 & 2 & & 9 \\
\hline 6 & 4 & 4 & 0 & 2 & & 3 \\
\hline 7 & 3 & 1 & 3 & 3 & & 2 \\
\hline 8 & 4 & 3 & 2 & 2 & & 3 \\
\hline 9 & 3 & 5 & 3 & 2 & & 1 \\
\hline 10 & 3 & 5 & 3 & 3 & & 1 \\
\hline 11 & 3 & 5 & 3 & 4 & & 1 \\
\hline 12 & 4 & 6 & 4 & 4 & & 1 \\
\hline 13 & 6 & 3 & 0 & 2 & & 7 \\
\hline 14 & 4 & 2 & 1 & 2 & & 1 \\
\hline 15 & 6 & 2 & 2 & 3 & & 1 \\
\hline 16 & 4 & 4 & 2 & 2 & & 1 \\
\hline 17 & 6 & 4 & 2 & 2 & & 2 \\
\hline 18 & 3 & 6 & 2 & 2 & & 2 \\
\hline 19 & 4 & 6 & 2 & 2 & & 1 \\
\hline 20 & 4 & 2 & 2 & 3 & & 2 \\
\hline 21 & 8 & 7 & 2 & 3 & $\begin{array}{l}\text { E. carotovora subsp. betavasculorum/ } \\
\text { E. rhapontici }\end{array}$ & $9 / 2$ \\
\hline 22 & 9 & 3 & 7 & 5 & E. carotovora subsp. odorifera & 12 \\
\hline 23 & 11 & 6 & 6 & 3 & E. carotovora subsp. wasabiae & 5 \\
\hline 24 & 13 & 2 & 2 & 7 & E. cypripedii & 3 \\
\hline 25 & 10 & 10 & 8 & 6 & E. ananas/E. uredovora & $3 / 1$ \\
\hline 26 & 10 & 10 & 2 & 6 & E. ananas & 1 \\
\hline 27 & 18 & 0 & 0 & 1 & E. herbicola & 2 \\
\hline 28 & 21 & 6 & 0 & 3 & E. herbicola/E. milletiae/Pantoea sp. & $4 / 1 / 1$ \\
\hline 29 & 22 & 9 & 2 & 3 & E. cacticida & 1 \\
\hline 30 & 23 & 9 & 2 & 3 & & 8 \\
\hline 31 & 24 & 9 & 2 & 3 & & 6 \\
\hline 32 & 12 & 1 & 2 & 3 & E. amylovora & 9 \\
\hline 33 & 16 & 2 & 0 & 2 & E. chrysanthemi & 3 \\
\hline 34 & 14 & 2 & 0 & 2 & & 3 \\
\hline 35 & 20 & 1 & 0 & 3 & & 6 \\
\hline 36 & 18 & 1 & 2 & 4 & & 2 \\
\hline 37 & 17 & 2 & 5 & 4 & & 1 \\
\hline 38 & 7 & 1 & 0 & 3 & & 2 \\
\hline 39 & 7 & 2 & 2 & 3 & & 1 \\
\hline 40 & 16 & 1 & 2 & 4 & & 1 \\
\hline 41 & 19 & 2 & 0 & 2 & & 1 \\
\hline 42 & 15 & 2 & 0 & 2 & & 1 \\
\hline 43 & 18 & 7 & 2 & 3 & & 1 \\
\hline 44 & 8 & 2 & 0 & 2 & & 1 \\
\hline 45 & 14 & 3 & 2 & 2 & & 1 \\
\hline 46 & 14 & 2 & 0 & 4 & & 1 \\
\hline 47 & 14 & 2 & 0 & 3 & & 1 \\
\hline 48 & 25 & 11 & 10 & 6 & E. stewartii & 2 \\
\hline 49 & 26 & 14 & 2 & 1 & E. persicina & 2 \\
\hline 50 & 29 & 8 & 2 & 2 & E. rubrifaciens & 1 \\
\hline
\end{tabular}


Table 2 (cont.)

\begin{tabular}{|c|c|c|c|c|c|c|}
\hline \multirow[t]{2}{*}{$\begin{array}{l}\text { RFLP } \\
\text { group* }\end{array}$} & \multicolumn{4}{|c|}{$\begin{array}{c}\text { PCR-RFLP patterns for specific restriction } \\
\text { endonucleases } \dagger\end{array}$} & \multirow[t]{2}{*}{ Erwinia species assigned } & \multirow[t]{2}{*}{$\begin{array}{l}\text { No. of } \\
\text { strains }\end{array}$} \\
\hline & TasI & $\operatorname{Tru} 1 \mathrm{I}$ & $A l u \mathrm{I}$ & HinfI & & \\
\hline 51 & 28 & 0 & 0 & 2 & E. nigrifluens & 1 \\
\hline 52 & 27 & 1 & 6 & 3 & E. salicis & 1 \\
\hline 53 & 30 & 14 & 2 & 3 & E. rhapontici & 1 \\
\hline 54 & 31 & 13 & 9 & 3 & E. psidii & 1 \\
\hline 55 & 0 & 6 & 9 & 3 & E. mallotivora & 1 \\
\hline 56 & 17 & 12 & 10 & 3 & E. quercina & 1 \\
\hline 57 & 23 & 9 & 2 & 1 & E. tracheiphila & 1 \\
\hline
\end{tabular}

* Numbers of RFLP groups based on the combined PCR-RFLP patterns. Groups were restricted to one species, except groups 21, 25 and 28.

† Numbers correspond to RFLP patterns shown in Fig. 1. Zero indicates the absence of restriction digestion by a given endonuclease.

found in this study between RFLP groups and host plants or geographical origins of the E. carotovora subsp. carotovora and E. chrysanthemi strains tested. The apparent genetic homogeneity in E. amylovora and E. carotovora subsp. atroseptica could have been magnified by the fact that both bacteria were probably relatively recently distributed across the world from their centres of origin, E. amylovora from North America in the 20th century (Schroth et al., 1974) and E. carotovora subsp. atroseptica from South America in the 16th century (Salaman, 1949). Most of the strains of these species studied in this paper originated from countries other than the centre of origin.

In contrast to E. amylovora, E. cacticida, E. chrysanthemi, E. carotovora subsp. atroseptica, E. carotovora subsp. betavasculorum, E. carotovora subsp. carotovora and E. carotovora subsp. odorifera, only a small number of strains of other species have been examined in this study. This is because only a few strains are listed in culture collections and those available tend to have been isolated usually from one host plant and from one geographical area. Therefore, the presence of only a few groups per species does not necessarily signify that the species are genetically homogeneous. Only when more strains from a wider range of sources have been studied can this point be verified.

In conclusion, the recA PCR-RFLP method can be used to rapidly identify species and subspecies of pectinolytic Erwinia. It allowed identification of all of the species of the former Erwinia genus, including members of the genus that have not been well studied and are not identified by traditional methods.

In addition, the method is a useful tool to study species diversity in relation to host specificity, host range and geographical distribution. It would also be useful to examine the distribution of bacteria connected with the worldwide exchange of plant material.

\section{ACKNOWLEDGEMENTS}

The authors wish to thank all of the scientists whose names are listed in the footnote to Table 1 for providing Erwinia strains. Special thanks are addressed to Dr M. C. M. Perombelon, Dr J. M. van der Wolf and Professor K. Geider for reading of the manuscript and helpful comments and suggestions. This work was supported by the Polish Scientific Committee (KBN) Grant 6 PO4B 01518 and PPH ABO which contributed some chemicals by donation.

\section{REFERENCES}

Alcorn, S. M., Orum, T. V., Steigerwalt, A. G., Foster, J. L. M., Fogleman, J. C. \& Brenner, D. J. (1991). Taxonomy and pathogenicity of Erwinia cacticida sp. nov. Int J Syst Bacteriol 41, 197-212.

Ausubel, F. M., Brent, R., Kingston, R. E., Moore, D. D., Seidman, J. G., Smith, J. A. \& Struhl, K. (editors). (1992). Short Protocols in Molecular Biology, 2nd edn. New York: Greene Publishing Associates and Wiley.

Beji, A., Mergaert, J., Gavini, F., Izard, D., Kersters, K., Leclerc, H. \& De Ley, J. (1988). Subjective synonymy of Erwinia herbicola, Erwinia milletiae, and Enterobacter agglomerans and redefinition of the taxon by genotypic and phenotypic data. Int J Syst Bacteriol 38, 77-88.

Bereswill, S., Pahl, A., Bellemann, P., Zeller, W. \& Geider, K. (1992). Sensitive and species-specific detection of Erwinia amylovora by PCR-analysis. Appl Environ Microbiol 58, 3522-3526.

Bereswill, S., Bugert, P., Bruchmüller, I. \& Geider, K. (1995). Identification of the fire blight pathogen, Erwinia amylovora, by PCR with chromosomal DNA. Appl Environ Microbiol 61, 2636-2642.

Blackwood, K. S., He, C., Gunton, J., Turenne, C. Y., Wolfe, J. \& Kabani, A. M. (2000). Evaluation of recA sequences for identification of Mycobacterium species. J Clin Microbiol 38, 2846-2852.

Brenner, D. J., Fanning, G. R. \& Steigerwalt, A. G. (1974). Deoxyribonucleic acid relatedness among Erwiniae and other Enterobacteriaceae: the gall, wilt, and dry-necrosis organisms (Genus Erwinia Winslow et al., sensu stricto). Int J Syst Bacteriol 24, 197-204. 
Brenner, D. J., Fanning, G. R., Leete Knutson, J. K., Steigerwalt, A. G. \& Krichevsky, M. I. (1984). Attempts to classify herbicola group-Enterobacter agglomerans strains by deoxyribonucleic acid hybridization and phenotypic tests. Int J Syst Bacteriol 34, $45-55$.

Darrasse, A., Priou, S., Kotoujansky, A. \& Bertheau, Y. (1994). PCR and restriction fragment length polymorphism of a pel gene as a tool to identify Erwinia carotovora in relation to potato diseases. Appl Environ Microbiol 60, 1437-1443.

De Boer, S. H. \& McNaughton, M. E. (1987). Monoclonal antibodies to the lipopolysaccharide of Erwinia carotovora subsp. atroseptica serogroup I. Phytopathology 77, 828-832.

De Boer, S. H. \& Ward, L. J. (1995). PCR detection of Erwinia carotovora subsp. atroseptica associated with potato tissue. Phytopathology 85, 854-858.

Dye, D. W. (1968). A taxonomic study of the genus Erwinia. I. The 'Amylovora' group. N Z J Sci 11, 590-607.

Dye, D. W. (1969a). A taxonomic study of the genus Erwinia. II. The 'Carotovora' group. N Z J Sci 12, 81-97.

Dye, D. W. (1969b). A taxonomic study of the genus Erwinia. III. The 'Herbicola' group. N Z J Sci 12, 223-236.

Dye, D. W. (1969c). A taxonomic study of the genus Erwinia. IV. 'Atypical' erwinias. N Z J Sci 12, 833-839.

Eisen, J. A. (1995). The RecA protein as a model molecule for molecular systematic studies of bacteria: comparison of trees of RecAs and 16S rRNAs from the same species. J Mol Evol 41, 1105-1123.

Fréchon, D., Exbrayat, P., Helias, V. \& 10 other authors (1998). Evaluation of a PCR kit for the detection of Erwinia carotovora subsp. atroseptica on potato tubers. Potato Res 41, 163-173.

Gavini, F., Mergaert, J., Beji, A., Mielcarek, C., Izard, D., Kersters, K. \& De Ley, J. (1989). Transfer of Enterobacter agglomerans (Beijerinck 1888) Ewing and Fife 1972 to Pantoea gen. nov. as Pantoea agglomerans comb. nov. and description of Pantoea dispersa sp. nov. Int J Syst Bacteriol 39, 337-345.

Gorris, M. T., Alarcon, B., Lopez, M. M. \& Cambra, M. (1994). Characterization of monoclonal antibodies specific for Erwinia carotovora subsp. atroseptica and comparison of serological methods for its sensitive detection on potato tubers. Appl Environ Microbiol 60, 2076-2085.

Guilford, P. J., Taylor, R. K., Clark, R. G., Hale, C. N. \& Forster, R. L. (1996). PCR-based techniques for the detection of Erwinia amylovora. Acta Hortic 411, 53-56.

Hao, M. V., Brenner, D. J., Steigerwalt, A. G., Kosako, Y. \& Komagata, K. (1990). Erwinia persicinus, a new species isolated from plants. Int J Syst Bacteriol 40, 379-383.

Hauben, L., Moore, E. R. B., Vauterin, L., Steenackers, M., Verdonck, L. \& Swings, J. (1998). Phylogenetic position of phytopathogens within the Enterobacteriacae. Syst Appl Microbiol 21, 384-397.

Hyman, L. J., Wallace, A., Lopez, M. M., Cambra, M., Gorris, M. T. \& Pérombelon, M. C. M. (1995). Characterization of monoclonal antibodies against Erwinia carotovora subsp. atroseptica serogroup I: specificity and epitope analysis. J Appl Bacteriol 78, 437-444.

Karlin, S., Weinstock, G. M. \& Brendel, V. (1995). Bacterial classification derived from RecA protein sequence comparisons. $J$ Bacteriol 177, 6881-6893.

Kim, W. S., Gardan, L., Rhim, S. L. \& Geider, K. (1999). Erwinia pyrifoliae sp. nov., a novel pathogen that affects Asian pear trees (Pyrus pyrifolia Nakai). Int J Syst Bacteriol 49, 899-906.
Kwon, S. W., Go, S. J., Kang, H. W., Ryu, J. C. \& Jo, J. K. (1997). Phylogenetic analysis of Erwinia species based on 16S rRNA gene sequences. Int J Syst Bacteriol 47, 1061-1067.

Lelliott, R. A. \& Dickey, R. S. (1984). Genus VII. Erwinia Winslow, Broadhurst, Buchanan, Krumwiede, Rogers and Smith 1920. In Bergey's Manual of Systematic Bacteriology, vol. 1, pp. 469-476. Edited by N. R. Krieg \& J. G. Holt. Baltimore: Williams \& Wilkins.

Lind, E. \& Ursing, J. (1986). Clinical strains of Enterobacter agglomerans (synonyms: Erwinia herbicola, Erwinia milletiae) identified by DNA-DNA hybridization. Acta Pathol Microbiol Immunol Scand Sect B 94, 205-213.

Lloyd, A. T. \& Sharp, P. M. (1993). Evolution of the recA gene and molecular phylogeny of bacteria. J Mol Evol 37, 399-407.

Ludwig, W. \& Schleifer, K. H. (1999). Phylogeny of bacteria beyond the 16 rRNA standard. ASM News 65, 752-757.

McManus, P. S. \& Jones, A. L. (1995). Detection of Erwinia amylovora by nested PCR and PCR-dot-blot and reverse-blot hybridisations. Phytopathology 85, 618-623.

Mahenthiralingam, E., Bischof, J., Byrne, S. K., Radomski, C., Davies, J. E., Av-Gay, Y. \& Vandamme, P. (2000). DNA-based diagnostic approaches for identification of Bulkholderia cepacia complex, Bulkholderia vietnamiensis, Bulkholderia multivorans, Bulkholderia stabilis, and Bulkholderia cepacia genomovars I and III. J Clin Microbiol 38, 3165-3173.

Mergaert, J., Verdonck, L., Kersters, K., Swings, J., Boeufgras, J. M. \& De Ley, J. (1984). Numerical taxonomy of Erwinia species using API systems. J Gen Microbiol 130, 1893-1910.

Mergaert, J., Verdonck, L. \& Kersters, K. (1993). Transfer of Erwinia ananas (synonym, Erwinia uredovora) and Erwinia stewartii to the genus Pantoea emend. as Pantoea ananas (Serrano 1928) comb. nov. and Pantoea stewartii (Smith 1898) comb. nov., respectively, and description of Pantoea stewartii subsp. indologenes subsp. nov. Int J Syst Bacteriol 43, 162-173.

Mergaert, J., Hauben, L., Cnockaert, M. C. \& Swings, J. (1999). Reclassification of non-pigmented Erwinia herbicola strains from trees as Erwinia billingiae sp. nov. Int J Syst Bacteriol 49, 377-383.

Nassar, A., Darrasse, A., Lemattre, M., Kotoujansky, A., Dervin, C., Vedel, R. \& Bertheau, Y. (1996). Characterization of Erwinia chrysanthemi by pectinolytic isozyme polymorphism and restriction fragment length polymorphism analysis of PCR-amplified fragments of pel genes. Appl Environ Microbiol 62, 22282235.

Nowak, A. \& Kur, J. (1995). Genomic species typing of acinetobacters by polymerase chain reaction amplification of the recA gene. FEMS Microbiol Lett 130, 327-332.

Perombelon, M. C. M. \& Kelman, A. (1980). Ecology of the soft rot erwinias. Annu Rev Phytopathol 18, 361-387.

Salaman, R. (1949). The History and Social Influence of the Potato. Cambridge: Cambridge University Press.

Samson, R., Ngwira, N. \& Rivera, N. (1989). Biochemical and serological diversity of Erwinia chrysanthemi. In Proceedings of the 7th International Conference on Plant Pathogenic Bacteria, pp. 895-901. Edited by Z. Klement. Budapest: Akademidi Kiadó és Nyomoda Vállalat.

Schroth, M., Thomson, S. V. \& Hildebrand, D. C. (1974). Epidemiology and control of fireblight. Annu Rev Phytopathol 12, 389-412.

Smid, E. J., Jansen, A. H. J. \& Gorris, L. G. M. (1995). Detection of Erwinia carotovora subsp. atroseptica and Erwinia chrysanthemi in potato tubers using polymerase chain reaction. Plant Pathol 44, 1058-1069. 
Śledź, W., Jafra, S., Waleron, M. \& Lojkowska, E. (2000). Genetic diversity of Erwinia carotovora strains isolated from infected plants grown in Poland. EPPO Bull 30, 403-407.

Toth, I. K., Hyman, L. J. \& Wood, J. R. (1999a). A one step PCRbased method for the detection of economically important soft rot Erwinia species on micropropagated potato plants. J Appl Microbiol 87, 158-166.

Toth, I. K., Bertheau, Y., Hyman, L. J. \& 9 other authors (1999b). Evaluation of phenotypic and molecular typing techniques for determining diversity in Erwinia carotovora subsp. atroseptica. J Appl Microbiol 87, 770-781.

Vantomme, R., Swings, J., Goor, M., Kersters, K. \& De Ley, J. (1982). Phytopathological, serological, biochemical and protein electrophoretic characterization of Erwinia amylovora strains isolated in Belgium. Phytopathol Z 103, 349-360.

Verdonck, L., Mergaert, J., Rijckaert, C., Swings, J., Kersters, K. \& De Ley, J. (1987). The genus Erwinia: a numerical analysis of phenotypic features. Int J Syst Bacteriol 37, 4-18.

Waleron, M., Waleron, K. \& Łojkowska, E. (2001). Application of recA PCR-RFLP for differentiation of Erwinia carotovora associ- ated with potato plants in tuber. In Proceedings of the 10th International Conference on Plant Pathogenic Bacteria, pp. 385-388, Charlottetown, Canada. Dordrecht: Kluwer.

Watanabe, K. \& Sato, M. (1998). Detection of variation of the Rdomain structure of ice nucleation genes in Erwinia herbicolagroup bacteria by PCR-RFLP analysis. Curr Microbiol 37, 201-209.

Willson, W. J., Wiedmann, M., Dillard, H. R. \& Batt, C. A. (1994). Identification of Erwinia stewartii by a ligase chain reaction assay. Appl Environ Microbiol 60, 278-284.

Zhang, Y. \& Geider, K. (1997). Differentiation of Erwinia amylovora strains by pulsed-field gel electrophoresis. Appl Environ Microbiol 63, 4421-4426.

Zhao, X. \& McEntee, K. (1990). DNA sequence analysis of the recA genes from Proteus vulgaris, Erwinia carotovora, Shigella flexneri and Escherichia coli B/r. Mol Gen Genet 222, 369-376.

Received 15 May 2001; revised 28 September 2001; accepted 15 October 2001. 